\title{
IoT for ITS: A Dynamic Traffic Lights Control based on the Kerner Three Phase Traffic Theory
}

\author{
Rghioui Anass \\ Faculty of Sciences and \\ Technologies of Tangier, \\ LIST Laboratory, \\ Department of Computer \\ Science, Morocco
}

\author{
Hernafi Yassine \\ Faculty of Sciences and \\ Technologies of Tangier, \\ LIST Laboratory, \\ Department of Computer \\ Science, Morocco
}

\author{
Bouhorma Mohammed \\ Faculty of Sciences and \\ Technologies of Tangier, LIST \\ Laboratory, Department of \\ Computer Science, Morocco
}

\begin{abstract}
A traffic light is a device for the regulation of traffic between road users, vehicles and pedestrians. The setting and synchronization of traffic lights of an axis or an area are very complex, and sometimes unsatisfactory for all or part of them. In addition, according to several studies, traffic lights would be responsible for half of the traffic jams and thus half the pollution, and poorly regulated lights can cause the tripling of the fuel consumption, therefore $\mathrm{CO}_{2}$ emissions and other pollutants emissions, when traffic is cluttered or too sparse. In our solution, we will try to apply the Kerner three-phase traffic theory to realize a synchronized system by establishing an Intelligent Transport System that will provide automatic management of traffic lights, while establishing a communication mode based on the concept of the Internet of Things for various traffic lights controllers to enable them to collaborate. In order to resolve the traffic jam issues, so the reduce of $\mathrm{CO}_{2}$ emissions and also the mobility metrics like the travel time. This paper is part of a project entitled 'V2IoT' which aims to use the techniques and concepts of the Internet of Things to improve ITS and the vehicular communications, namely, the V2V and the V2I systems within smart cities.
\end{abstract}

\section{Keywords}

Smart cities, Internet of Things, Intelligent Transportation Systems, Vehicular Ad Hoc networks.

\section{INTRODUCTION}

The concept of smart cities was founded there more than ten years, and since then a number of cities have taken the bandwagon and have qualified for "smart city" in one way or another. Rapid urbanization and the pressing need to develop a sustainable model for the long term to cope with the expected increase in the urban population [1].

Rapid urbanization poses a number of problems; policymakers must rethink and redefine the particular terms of infrastructure construction, supply of services, citizen participation and linkages between systems to ensure that cities become more sustainable and solid living spaces. City planners are shifting towards this integrated approach; they design the cities either as a set of distinct but as a global network. The aim is to offer people a better quality of life by combining technological and social innovations and the use of ICT to enhance the effectiveness of sectors such as transport, energy and quality of life.

Although the role of information and communications technology (ICT) is not yet fully defined, services that ICT can support, including management of water resources, energy efficiency and transport infrastructure, are themselves well known.
ICT infrastructure is the nervous system of any intelligent city; coordinates interactions between different components and physical infrastructure. It is the basis from which different intelligent services can operate efficiently and optimally.

One can consider the components of the physical infrastructure of a city like subnets of a larger network, such as subsystems within a system. These components are related to a network in terms of the characteristics of the final use and interactivity with other nodes, which are similar to those of a computer network or data communication.

On the other hand, IoT have attracted a lot of attention and our consideration to conceive the proposed approach. The evolution of the Internet of Things from a concept to a reality is considered as a big challenge for the combined scientific and technical teams that work on the development of the Internet [2]-[4]. It is an opportunity for those interested in technology and its employees as companies and developers, and all the rest of the other sectors, which will be used in order to develop their services and their products.

The number of embedded devices with IP is growing ascending continuously. It is expected to reach the 50 billion device by 2020 [5], due to the evolution in the electronics sector, processors, transmitters, communication component, and batteries, etc., what makes it easy to incorporate into many applications and machines, and even the invention of new ones that could not have been created by the past circumstances before this development.

The first objective of creating the Internet of Things and the development of its mechanisms is to make the devices more autonomous and independent from the direct control of the human user, and to allow this latter tracking and remote control these devices, each according to its field.

The remaining end of this evolution is to get more facilities and greater possibilities in making decisions. With the sensors, it became possible to obtain information and data directly from the physical world. Thanks to IP, they can be accessed from anywhere in the world in the real time, which give the opportunity to create intelligent devices (smart sensors). The collected data is used to make better and faster decisions. Sensors can now communicate with the other devices, surrounding it or in a distant, in order to exchange the necessary information.

To define conventionally the concept of Intelligent Transport Systems (ITS) [6], which referred to all services elaborated in order to enhance efficiency, quality for a pertinent infrastructure, we will focus on the application of Vehicular Ad hoc Networks (VANET) [7], [8] as a branch of Mobile networks [9]. The VANETs are characterized among other things by high dynamics due to the rapid mobility of vehicles. 
Vehicular network for intelligent transport systems that accredits to the application of new information technologies and transport communication, can help to improve road safety and traffic efficiency through the exchange of information between vehicles.

This type is characterized by a high mobility, in fact, the complexity of this network reside on the expected difference between the speeds of vehicles, which causes a permanent change due to intense rapid network topology. That said, we must adapt the mechanisms to be able to function effectively in this particular context. The different types of communication i.e. V2V (Vehicle to Vehicle) [10] this communication is established between two or more vehicles. This approach is more or less adapted to the vehicle networks short worn. The second type of communication is V2I (Vehicles to Infrastructure), communication is established between the equipment of the existing network infrastructure such as wireless access points and vehicles available in the range of equipment. These forms of communication offer a whole of safety services and applications to improve road safety, traffic efficiency and provide a green environment.

This paper proposed a smart communication entitled 'V2IoT' an improved intelligent transportation system offering the opportunity to exploit the benefits of smart mobility. This approach uses a mixture between the techniques and concepts of the Internet of Things and the types of vehicular communication systems within Smart Cities.

In short, the main of our contributions of the work are listed below:

- Dynamic traffic lights algorithm, which controlled the traffic signals.

- In order to deal with this sophisticated approach, we have introduced the suggested techniques to ensure fluidity and smooth traffic.

- We use a realistic VANET mobility traffic taken from the metropolitan area of Casablanca (Morocco), beneficial to dissect the results of simulation.

This paper is organized as follows. In the next section, the issues related to context considered are introduced. Section 3 and 4 describe the proposed approach to tackle the problem. Section 5 presents the experiments carry out, and an interpretation of results is shown in section 6. Finally, conclusions and perspectives are drawn in section 7 .

\section{CONTEXT OF TRAFFIC PROBLEMS}

A traffic jam or congestion routinely designates an embarrassment, congestion of traffic, which results in slower speeds usually or almost zero or by an irregular flow. This applies not only to the road sector, but also to pedestrians, rail vehicles, or in a broader field to signify congestion.

For several decades, the number and extent of congestion are generally increasing, especially in developing countries. The causes are many, the main one being the increase in car traffic (itself due to the increase of population and its automobile equipment rates). The poor state of certain infrastructures also promotes traffic jams and lack of users of information (which decreases thanks to information on real-time traffic).

As consequences, traffic jams have many economic, social, health and environmental consequences. In addition to reducing traffic speed, traffic jam is a major source of air pollution due to additional fuel consumption and less mixing of air layers with health costs and medium- and long-term climate through emissions of greenhouse gases.

When traffic jams prolong the journey, the vehicle uses more fuel. Studies show that the plugs can cause $\mathrm{CO}_{2}$ emissions by over 850 tones per year, and in one place. Fuel consumed when a vehicle is stopped in traffic results in the emission of greenhouse gases and pollutants resulting in deterioration in air quality. It should be stated beforehand that not all the traffic jams are equal, and there are consequently different consumption degrees.

In 2014, Inrix and the Centre for Economics and Business Research (Cebr) published a study [11] about the evolution of the economic and environmental cost of congestion in Europe and the US over the next 15 years.

For example, in France, transport accounts for $27 \%$ of emissions of greenhouse gases and $17 \%$ of energy consumption. Between 2013 and 2030, CO2 emissions are expected to increase by $13 \%$ in France. The financial amount of French $\mathrm{CO} 2$ emissions expected to increase from 11 million euros to 232 million euros between 2013 and 2030 due to substantial increases in the European market in $\mathrm{CO} 2$ emissions.

By 2030, the study predicts that worldwide, the caps will cost 221 billion euros per year to Western economies representing 70 billion euros more than in 2013 (151 billion euros). Of the four countries considered, France should see the third largest increase in the annual cost, with a bill that will increase from 17 billion euros in 2013 to 22 billion euros in $2030(+31 \%)$, behind the United Kingdom $(+63 \%)$ and the US $(+50 \%)$.

The increase in attendance of road networks is mainly due to lower cost of vehicle ownership, improved living standards, and a strong demand for car travel and to a population that continues to grow. Moreover, every year, traffic increases exponentially with the development of urban areas, making our infrastructure and inadequate management practices.

According to several studies, traffic lights would be responsible for half of the traffic jams and thus half the pollution, and poorly regulated lights can cause the tripling of the fuel consumption, therefore $\mathrm{CO}_{2}$ emissions, when traffic is cluttered or too sparse [12].

Today, these congestion problems related to the growing transportation within and between cities have become a global priority. The collective awareness of pollution on the environment and on citizens' quality of life results in an abundance of projects and initiatives such as the Smart Cities.

Many ways exist to fight against congestion, there may be mentioned: improving traffic flow, including better signage regulation of the use through pricing by using intelligent transport system. This requires the use of technology to manage the flow of traffic through the establishment of an intelligent transport system by using the concept and the technology of the Internet of Things.

\section{V2IoT APPROACH}

The theory of the three phases of the traffic is an alternative theory, modeling of traffic developed by Boris Kerner between 1996 and 2002 [13], [14]. Kerner model characterizes the traffic running as one of three possible schemes. Within each system, there are numerous configurations for growth or as the decline in the configuration, the location, the existence of severe congestion compared to a slight congestion and its relationship to other configurations. This approach is a quantitative approach that 
is limited to describing what is happening on the road. Allowing the identification of significant and recurring traffic patterns, this approach has the merit of being useful to monitor and prevent traffic congestion. It focuses primarily on the physical explanation of the deterioration of traffic conditions and congestion. Unlike classical theories based on the fundamental diagram of traffic that distinguish two phases or regimes (fluid traffic and congestion), the Kerner theory proposes three phases. It distinguishes in fact, two systems of congestion, the synchronized rate and major congestions and mobile, bringing to three the total number of plans: The fluid regime $(\mathrm{F})$, the synchronized regime $(\mathrm{S})$, major congestions and mobile $(\mathrm{J})$. A phase or regime is defined as a condition in time and in space.

The Fluid regime $(\mathrm{F})$, a positive correlation appears empirically between flow q (number of vehicles per time interval) and the k density (vehicles per unit of space). This relationship holds up to an upper limit corresponding to the critical density $K_{\text {crit }}$ (Fig. 1).

In the Congested regime, the vehicle speed is less than the minimum speed $V_{\text {free }}^{\text {min }}=\frac{q_{\max }}{k_{\text {crit }}}$ in fluid regime and the right having this value for slope (Blue line in Fig. 1) divides all the empirical data on the flow chart-density into two regions: fluid regime observations left and right congested regime.

In The Major mobile congestion ( $\mathrm{J})$, the congestion is a "major" if its width (in the direction of flow) clearly exceeds the width of his forehead. The average vehicle speed is much lower than that in the fluid system. Downstream from the front, the vehicles accelerate to speed in fluid regime. Upstream, the vehicles leave the fluid or synchronized regime and shall reduce speed. The average speed of the downstream front remains to $V_{g}$, even if the front propagates through other schemes of traffic or tightening of the road. The flow is drastically reduced in such congestion.

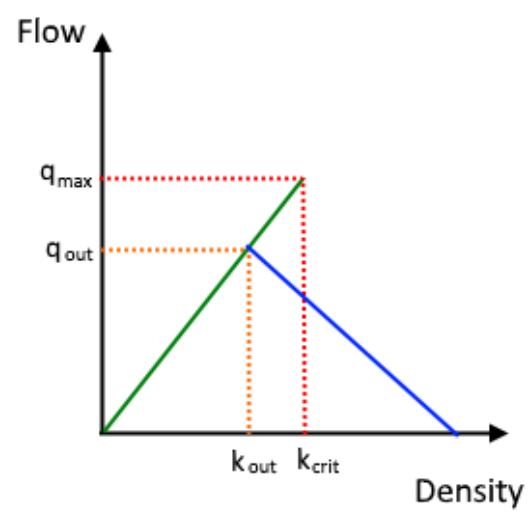

\section{Fig 1: Observations of the flow according to the density of fluid systems and congested}

However, the Synchronized regime (S), unlike major congestions, flow and speed can vary significantly in the scheme. The downstream front is generally spatially localized, usually in a narrowing of the road. The flow rate may remain equivalent to the fluid system, even if the speed is drastically reduced.

Since this synchronized flow has not the characteristics of a major congestion $\mathrm{J}$, the theory of the three phases of Kerner is assumed that homogeneous state of synchronized flows cover a region in two dimensions on the flow density diagram (Blue area in Fig. 2).

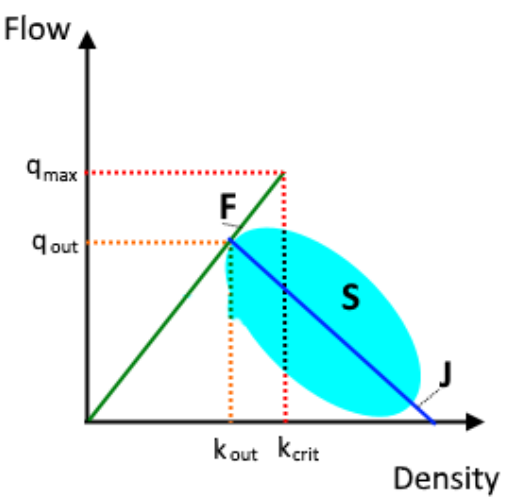

Fig 2: The three phases of the flow-density chart

We are interested in the definition of the synchronized regime to establish our solution of an automatically and dynamically traffic light system that allows a better traffic management.

A traffic light is a device for the regulation of traffic between road users, vehicles and pedestrians. The setting and synchronization of traffic lights of an axis or an area are very complex, and sometimes unsatisfactory for all or part of them.

The use of traffic lights should be limited to the regulation of traffic and on certain routes, the driving comfort of the users.

The problem with traditional traffic lights is that they alternate the same time for all the traffic lights across the road, although a denser than the other.

Sometimes in two crossroads, one of the two is the primary and the other is secondary, most of the time the number of vehicles driving is much lower, sometimes it's zero vehicle in a period. The traditional traffic light does not have the elements to handle this situation, so it puts the green light to this road if there is no vehicle, and blocks the other way. Simply because the controller allocates time alternately regardless of the situation of the road. Another problem, especially in the boulevards that stretch traffic for hundreds of meters. The vehicle has to stop several times while traveling on this boulevard, it will be better to give it the possibility to circulate continuously without stopping.

At rush hours, it creates many jams; a vehicle will be stuck in a multitude of traffic along the road. Due to this lack of communication between controllers running traffic lights, each of which works independently of the others. Even if we try to schedule them to be synced, this synchronization does not give good results since the passage of time of a vehicle between the first traffic light and the second depends on several factors, the most important of these elements is the speed of the vehicle according to the density of the road.

Our solution is to use a wireless sensor network interconnected calculating the density of a road and transfer these data to the base station placed at a traffic light controller level. Automatically and intelligently, the controller gives priority to the densest road to release by burning green to allow vehicles to circulate. Thus, the controllers are connected to each other via the Internet, so they can communicate and exchange information on traffic, and even direct vehicles to the less dense routes in the event of a congested traffic jam. 
The algorithm 1 defines the dynamic traffic light simulation algorithm which controls the traffic lights by using the concept of calculating the vehicle density reposed on the Kerner three phase traffic theory.

\section{PROPOSED SOLUTION}

In the Kerner method, we find the definition of three recognition systems of road conditions: fluid regime $(\mathrm{F})$, congested system (J) and synchronized system (S). Our solution is based on the definition of the synchronized system. We will compare the condition of two intersecting roads A and $\mathrm{B}$, the road is saying is in the congested state, taking A, will override the green light, until it becomes fluid. When the other road, in our example, B, becomes congested, it will override the green light until it becomes fluid, and vice-versa. The Fig. 3 shows the roads take into account to define the VANET urban scenario for our approach.

As in the method of Kerner, it was based on the calculation of two parameters that indicate the traffic condition, the density and the flow, these two parameters define the boundaries between a fluid traffic and congested traffic as explained in the overview.

We want by our method to achieve a fast and instant solution that will help us to determine the condition of a traffic mainly based on the infrastructure, without that vehicle will be equipped with sophisticated technologies, the reference scenario is depicted in Fig.4.

In the practical part, the sensors installed on the edges of two intersecting roads measure both parameters data, these data are transferred to the traffic light controller that are at the crossroads. It calculates in the time that data and determine the condition of the two roads, it will turn on the green light for the road is in the congested state and turns on the red light to the other, waiting for the other is found in the congested state.

In this case, it is necessary to differentiate between a congested system and congested major system using the method of Kerner, because the goal of our system is to avoid as much as possible the major congested state.

One problem is that if a long road, like a boulevard, is divided into several slices (one slice is bounded by red lights), if we will release the first slice leaving cars circulated to other slices, we will quickly find in a congested major system. The coming vehicles will accumulate with the vehicles whose are already in the second slice, waiting that the sensors take measurements and send the data to the controller to detect a state of congestion. For that, we proposed the communication between controllers to exchange information with each other, and they can prevent the condition of the road before it will be congested.

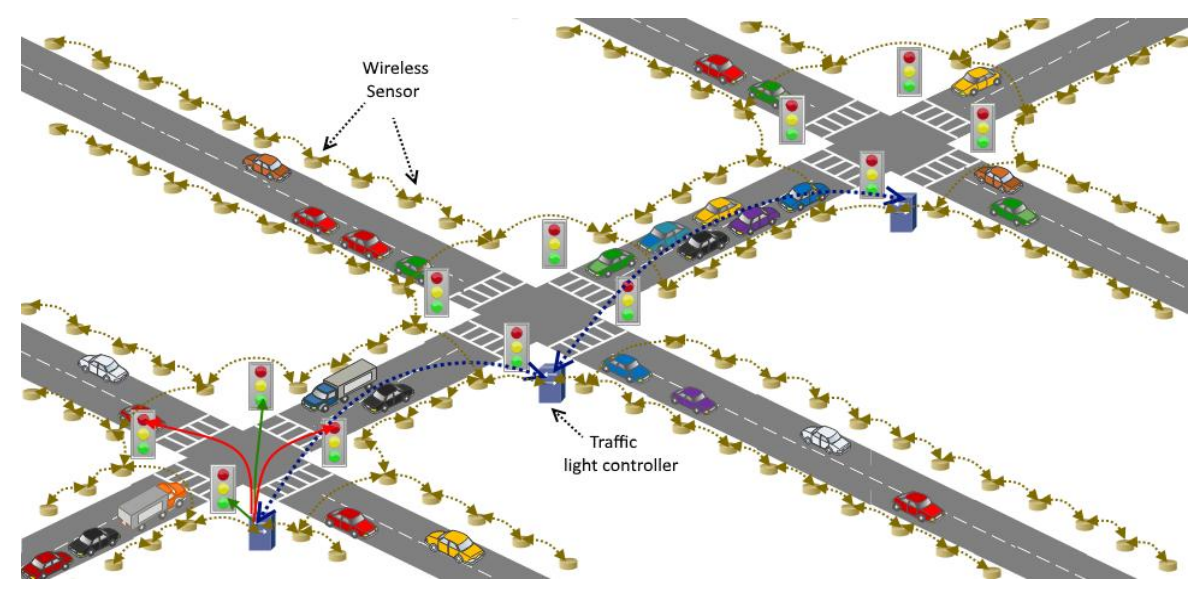

Fig. 3: The heterogeneous architecture for vehicular communications

By hydrodynamic analogy, the average speed of a stream of vehicles is defined as the ratio:

$V=\frac{q}{k}$

Where $k$ is the density and the flow rate $q$ (Fig.1.). For a flow rate of $2000 \mathrm{v} / \mathrm{h}$ and a density of 50 vehicles $/ \mathrm{km}$, the average speed of flow is $2000 / 50=40 \mathrm{~km} / \mathrm{h}$. However, if $k$ is small, $u$ is high, if $k$ increases, decreases $u, u$ suddenly the average speed is a monotonically decreasing function of the density $k$ :

$v=v(k)$

Then, we have just to determine the critical density threshold $k_{c}$ to determine whether traffic is flowing or congested:

$k<k_{\text {crit }}$ Fluid traffic

$k>k_{\text {crit }}$ Congested traffic

This part will be calculated for each slice, that being said for us will be just the congested state where vehicles will be waiting until the fire is green, but basically in a general way in its entire length, the system ensures that the flow is in the synchronized system.
In general, in our solution, we try to keep the system in the synchronized regime, for the reason that in the route in its full length in cities, it is impossible to keep the traffic in the fluid system since there are stops in traffic lights in cross roads, for balance, we must alter the priorities of traffic lights across.

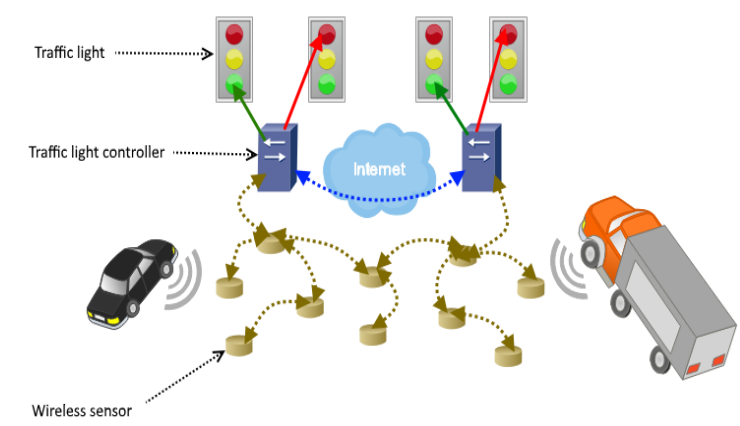

Fig. 4: The reference architecture scenario in urban traffic 


\section{SIMULATION PARAMETERS}

The main objective of these experiments is to evaluate our architecture and approach parameters in real traffic. We dissect the effects that these classes will have on traffic performance, related to the travel time and the environment. We have an intuition that traffic congestion cannot only affect road safety, but also the environment such as an increase in fuel consumption, pollutant emissions, the level of traffic noise, and also the quality of life

In this phase, we anticipate our approaches conceived by means of simulation experiments. The analysis will be reposed on SUMO (Simulation of Urban Mobility) the open source simulator refers to urban mobility. It allows the user to build a custom route topology, and also it helps to import different card formats in many cities worldwide. Also, in order to calculate pollutant emissions, we use the EMIT model a statistical model for instantaneous emissions and fuel consumption of vehicles integrated in SUMO.

Beneficial for the evaluation, the main metrics used in this work are cataloged as follows: the Average Travel Time, the total and the average waiting time, the average of vehicular travel length, the Fuel Consumption, Carbon Dioxide $\left(\mathrm{CO}_{2}\right)$, Particulate Matter $(P M)$, Nitrogen Oxides $\left(\mathrm{NO}_{x}\right)$, Carbon Monoxide $(\mathrm{CO})$, HydroCarbons $(\mathrm{CH})$, the noise emissions. In order to satisfy the approach, we can clearly distinguish that the ultimate ambition of this work consists to improve the QoS (Quality of Service)

Nevertheless, we applied a realistic scenario for traffic simulations taken from freely digital maps (the Open Street Map as described in Fig.5), by considering the important highway in the Casablanca city, Morocco and taking into account traffic lights, reel road directions, etc.

As a next step, the Fig.6, 7, 8 and 9 define respectively the procedure to generate a real traffic simulation, since the capabilities to import road networks from OpenStreetMap until the configuration and visualization of the simulations. The main parameters of the simulation are summarized in Table 1. In addition, Table 2 represents all the statistics reposed on results of this simulation of Casablanca city. We can depict that the average performances of vehicles for these trades are significantly different, the increased traffic load influences them, and the measures tend to rise when the traffic increases. To study the impact of the network density on the performance of the protocols for the assessment criteria, we conducted several simulations each time increasing the number of vehicles to describe a network with high mobility.

Table 1. Simulation parameters

\begin{tabular}{l|l}
\hline Parameter & Value \\
\hline & V2I/ V2V/ V2IoT \\
Communications & $802.11 \mathrm{p}$ \\
Mac Layer & 12 \\
Traffic logics & Two Ray Ground Model \\
Radio propagation & $2000 \mathrm{mx} 2000 \mathrm{~m}$ \\
Simulated area & $60 \mathrm{~km} / \mathrm{h}$ \\
Maximum speed & $1.4 \mathrm{~m} / \mathrm{s}^{2}$ \\
Maximum acceleration & $2.0 \mathrm{~m} / \mathrm{s}^{2}$ \\
Maximum deceleration & $1 \mathrm{~s}$ \\
Driver reaction time & 0.5 \\
Politeness factor & $1000 \mathrm{~s}$ \\
Simulation time & OSM (Open Street Map) \\
Map type & $95 \%$ \\
Confidence interval & 100 to 1000 \\
Number of vehicles & Random \\
Movement type & From 10 to $950 \mathrm{~s}(1000 \mathrm{~s})$ \\
Transmission time &
\end{tabular}

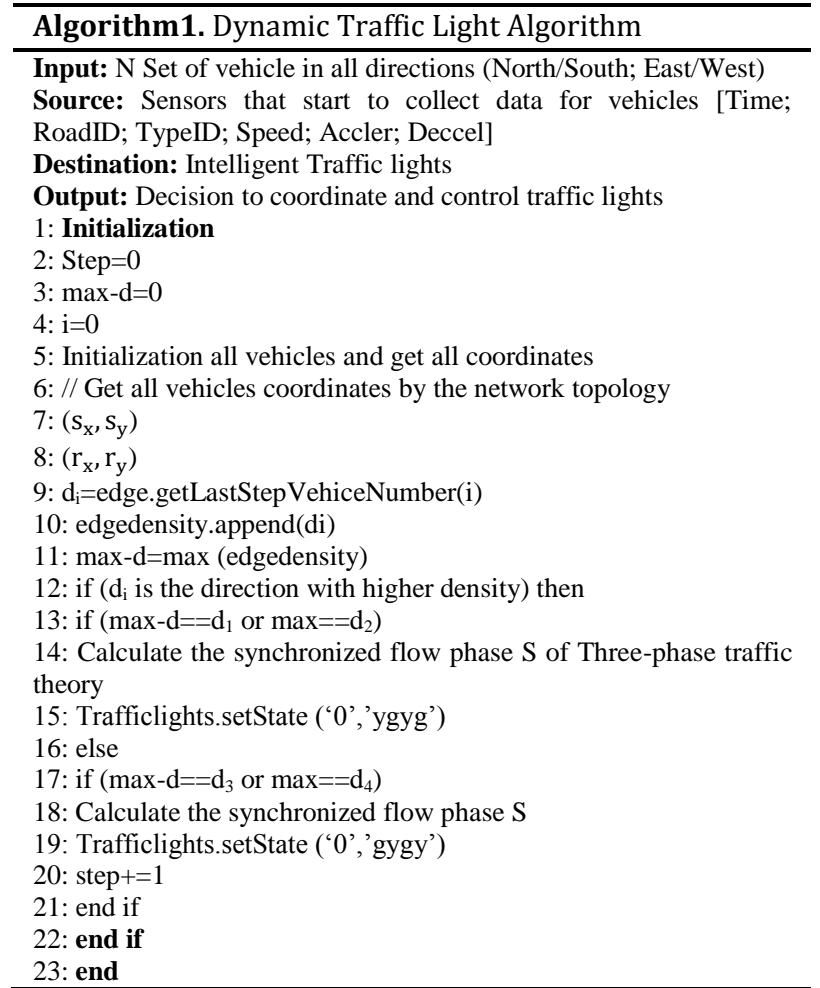

Table 2. Traffic statistics via Sumo

\begin{tabular}{l|llll}
\hline $\begin{array}{l}\text { Total Number of } \\
\text { Vehicles }\end{array}$ & 100 & 300 & 700 & 1000 \\
\hline $\begin{array}{l}\text { Total Departure Delay } \\
\text { (s) }\end{array}$ & 2 & 11 & 29 & 71 \\
$\begin{array}{l}\text { Total Waiting Time (s) } \\
\text { Average Vehicular }\end{array}$ & 218 & 2857 & 39696 & 61040 \\
$\begin{array}{l}\text { Waiting Time (s) } \\
\text { Total Travel Time (s) }\end{array}$ & 14984 & 51870 & 176361 & 204842 \\
$\begin{array}{l}\text { Average Vehicular } \\
\text { Travel Time (s) }\end{array}$ & 159 & 183 & 213 & 266 \\
$\begin{array}{l}\text { Total Travel Length (m) } \\
\text { Average Vehicular }\end{array}$ & 217461 & 716726 & 1684442 & 2381261 \\
$\begin{array}{l}\text { Travel Length (m) } \\
\text { Average Vehicular } \\
\text { Travel Speed (m/s) }\end{array}$ & 2313 & 2532 & 2485 & 2548 \\
\hline
\end{tabular}

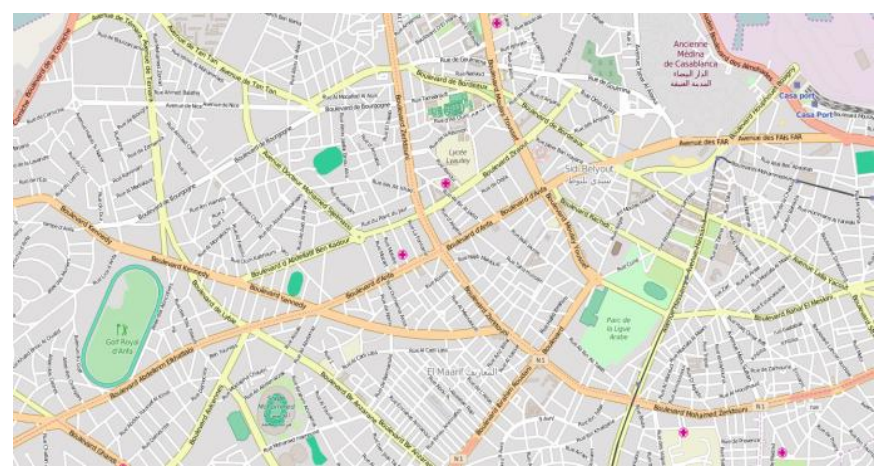

Fig. 5: Generalization of a real card of Casablanca (Morocco) using Open Street Map 


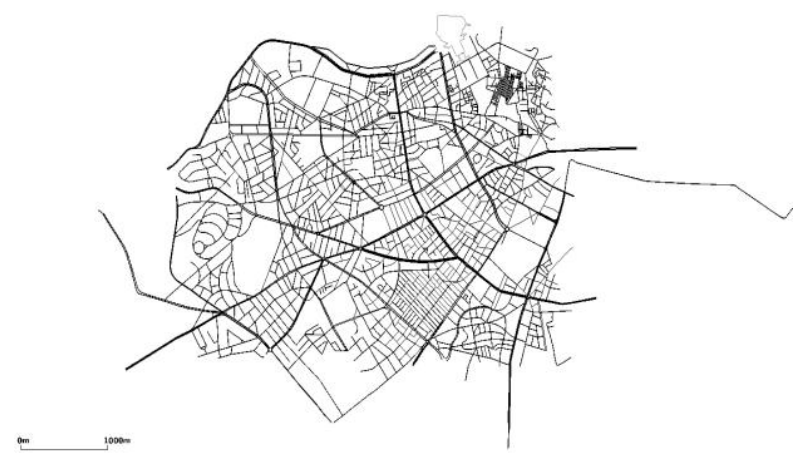

Fig. 6: Import the map of Casablanca (Morocco) using Open Street Map by the simulator SUMO

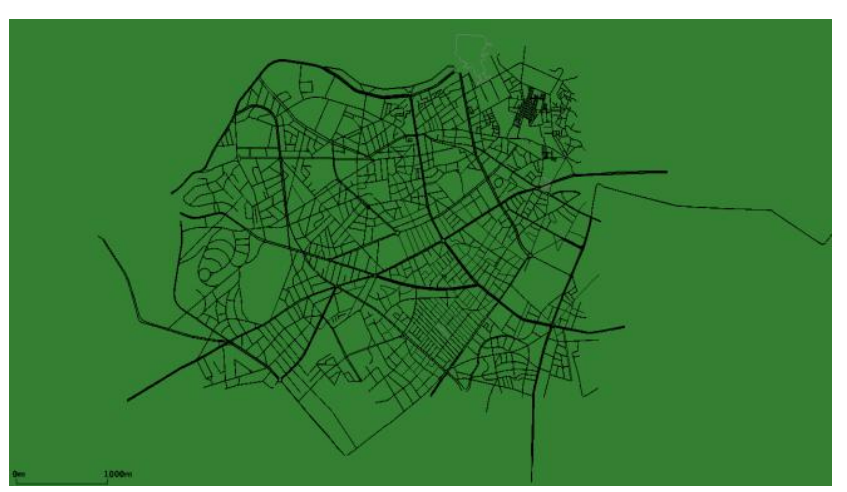

Fig. 7: Visualization of the real world of Casablanca (Morocco) by the simulator SUMO

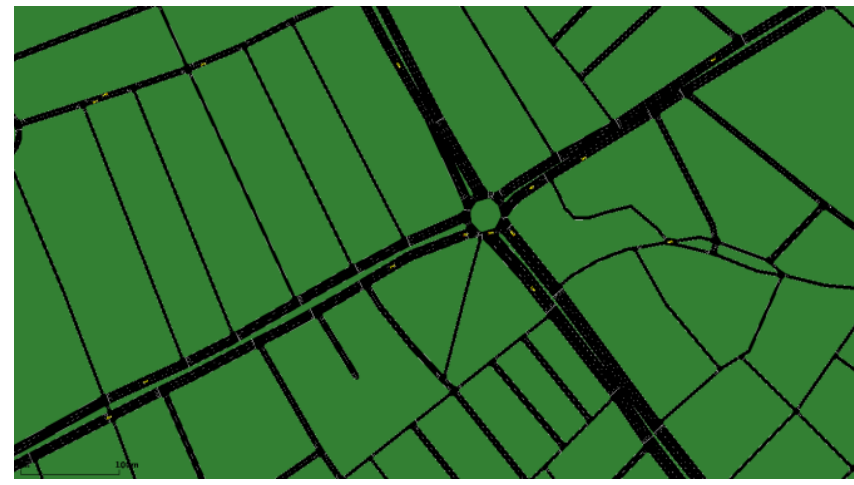

Fig. 8: Vehicle movements Visualisation of traffic roads

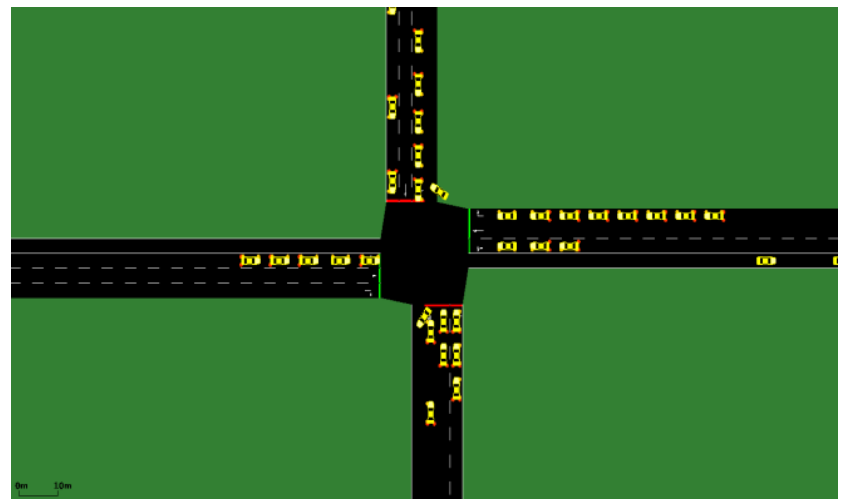

Fig. 9: Traffic lights Visualisation with the current state of traffic roads

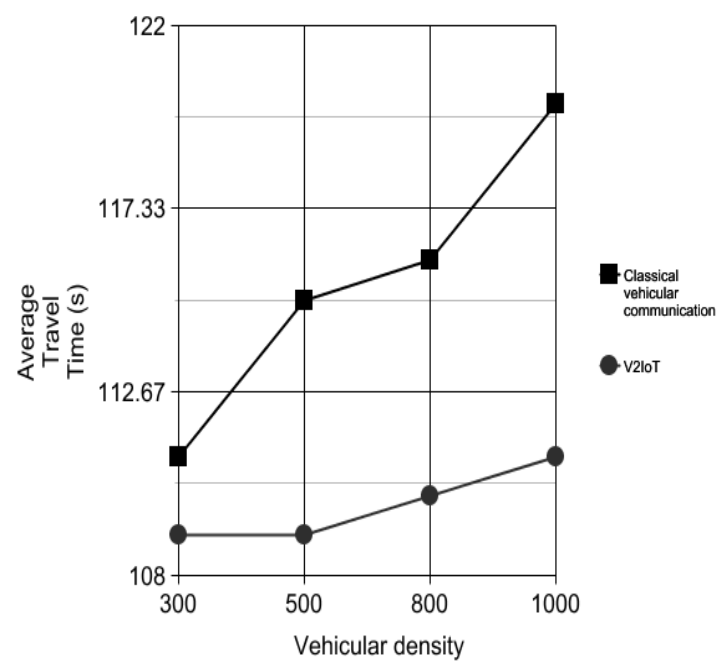

Fig. 10: Average travel time for classical vehicular communication and V2IoT, as a function of vehicle density

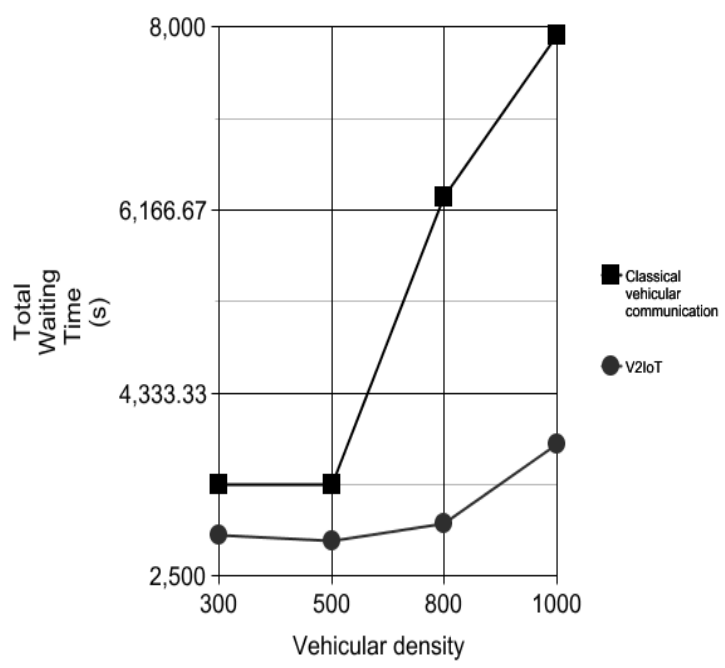

Fig. 11: Total waiting time for classical vehicular communication and V2IoT, as a function of vehicle density

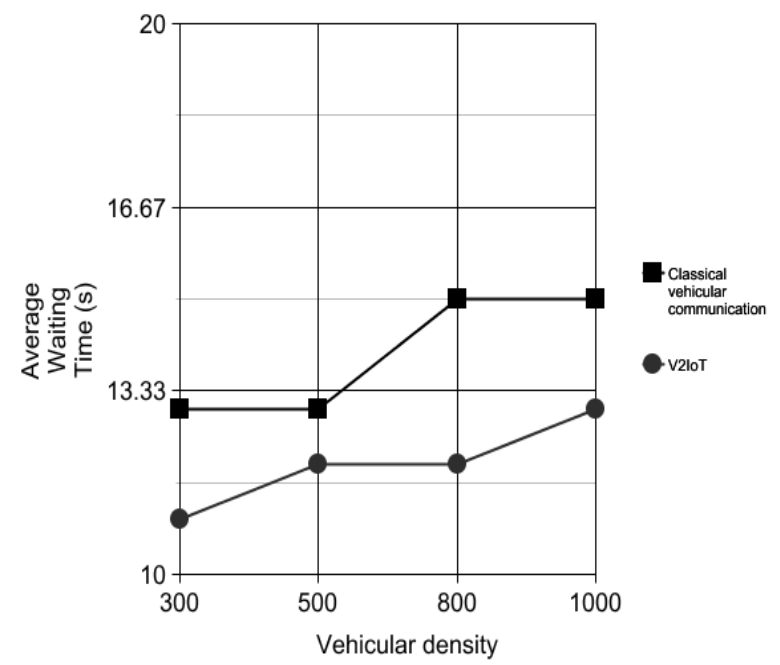

Fig. 12: Average waiting time for classical vehicular communication and V2IoT, as a function of vehicle density 


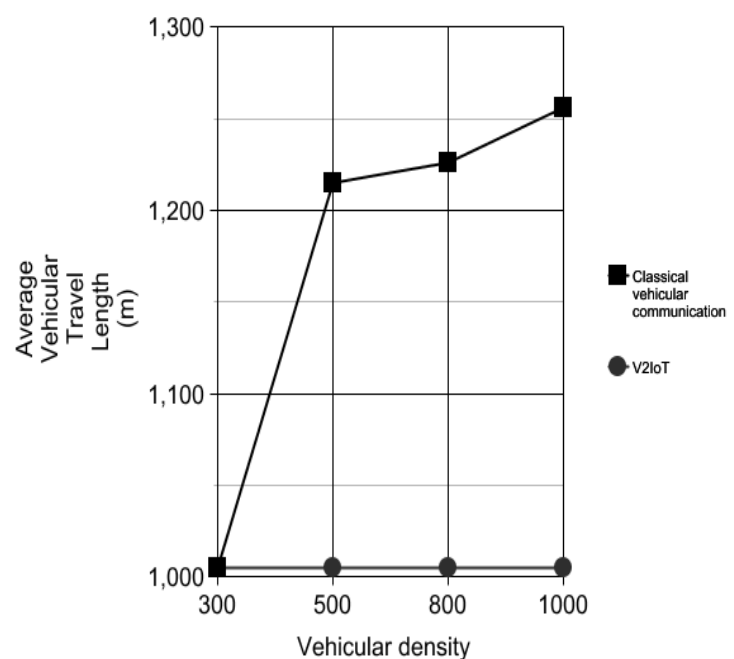

Fig. 13: Average vehicular travel length for classical vehicular communication and V2IoT, as a function of vehicle density

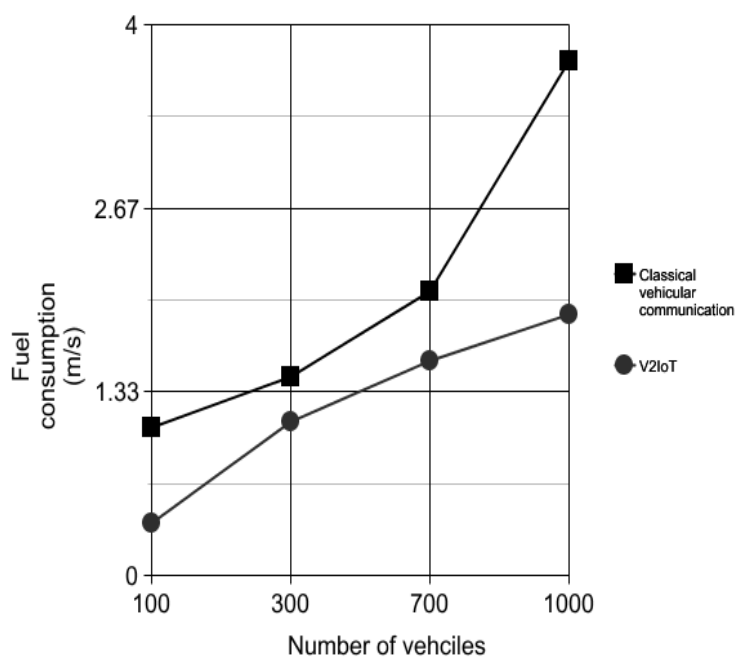

Fig. 14: Fuel consumption rate for classical vehicular communication and V2IoT, depending on vehicle density

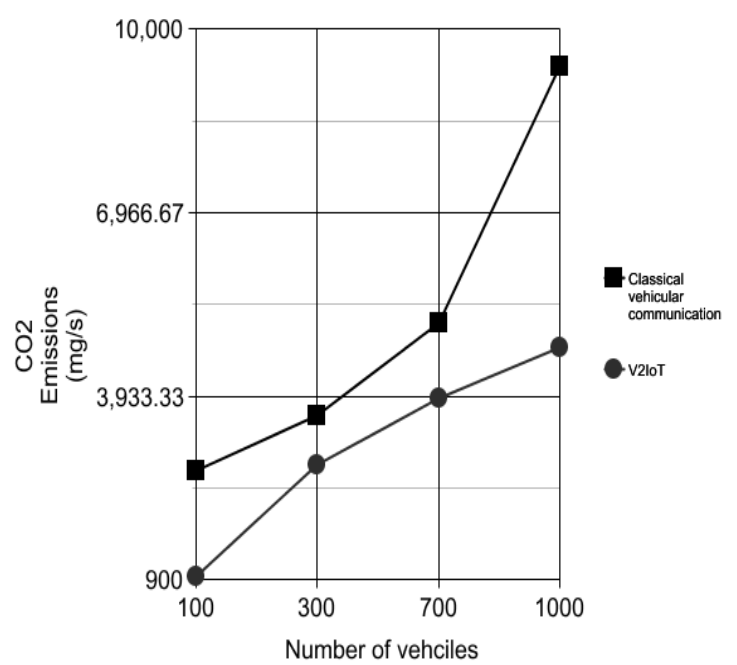

Fig. 15. $\mathrm{CO}_{2}$ Total emissions for classical vehicular communication and V2IoT, depending on vehicle density

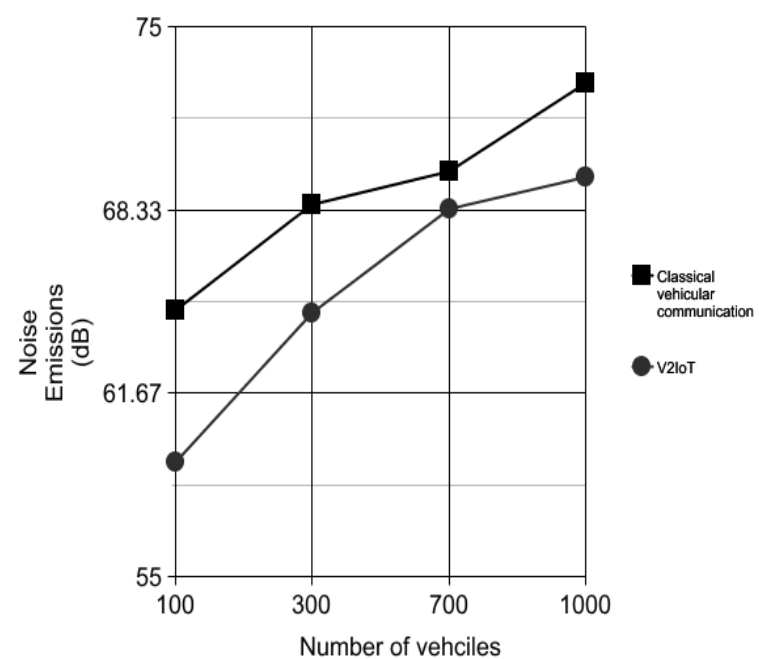

Fig. 16: The Noise emissions for classical vehicular communication and V2IoT, depending on vehicle density

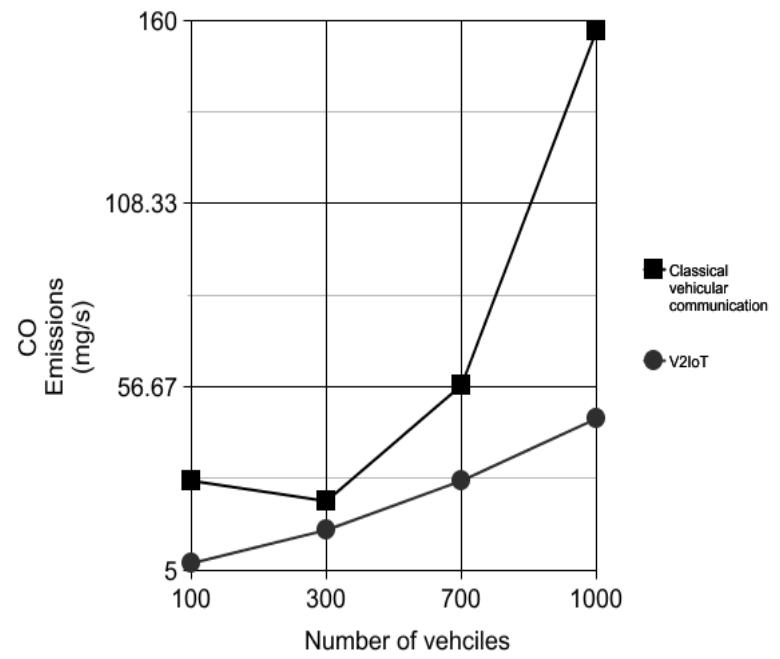

Fig. 17: The Carbon Monoxide (CO) emissions for classical vehicular communication and V2IoT, depending on vehicle density

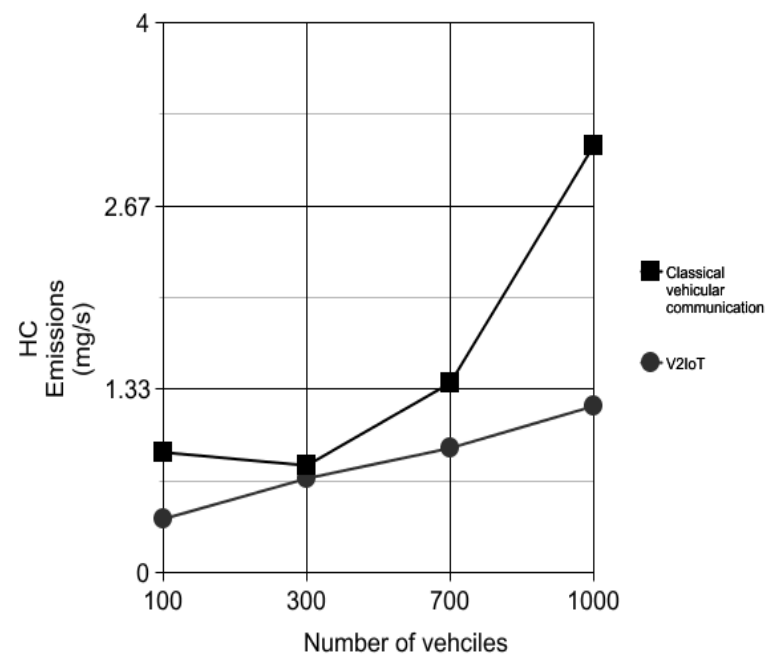

Fig. 18: The Hydrocarbons (HC) emissions for classical vehicular communication and V2IoT, depending on vehicle density 


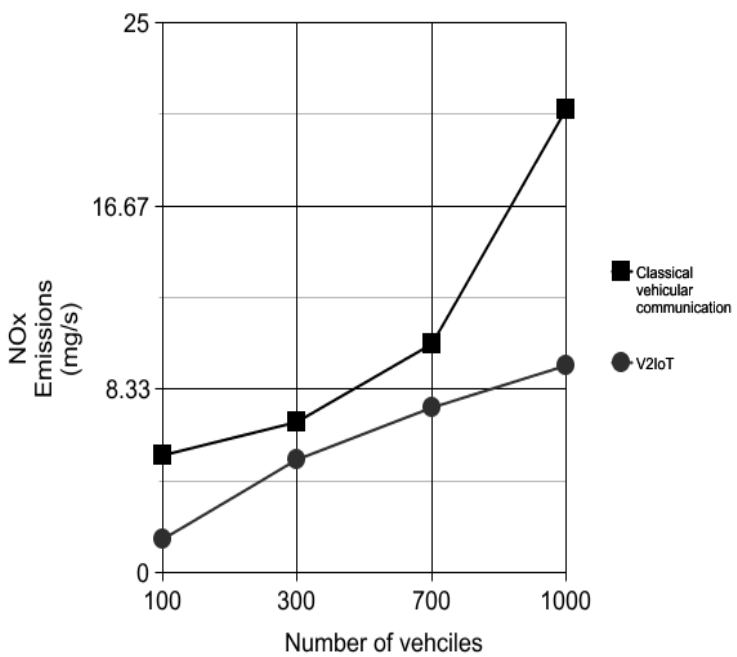

Fig. 19: The Nitrogen Oxides $\left(\mathrm{NO}_{\mathrm{X}}\right)$ emissions for classical vehicular communication and V2IoT, depending on vehicle density

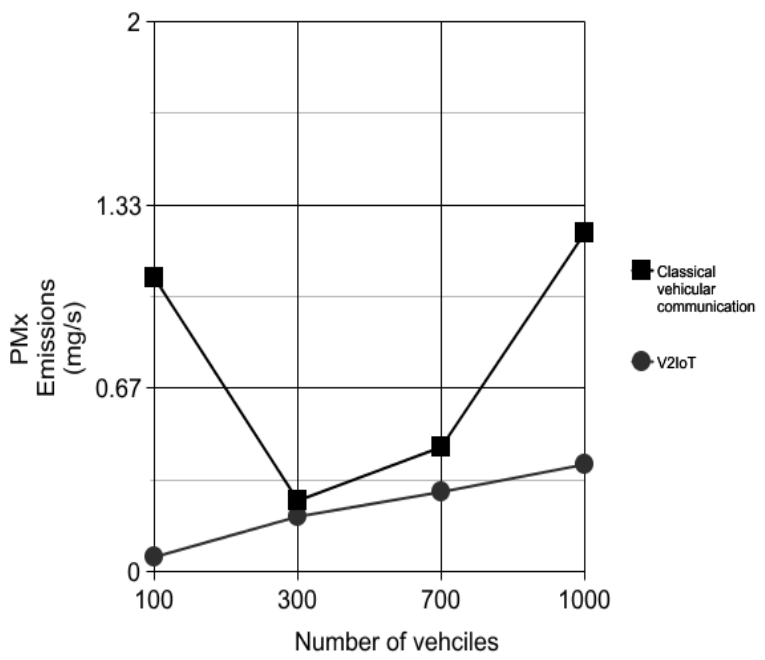

Fig. 20: The Particulate Matter (PM) emissions for classical vehicular communication and V2IoT, depending on vehicle density

\section{INTERPRETATION OF RESULTS}

We evaluated the average travel times in Fig. 9. based on the vehicle density in traffic. It's evident that the average travel time increases, when the approach of traffic congestion takes the front. However, our V2Iot approach was performing best results versus the classical communication of vehicular networks, which confirmed by a constant stability in comparison with the large raising measurements of average travel time in the opposite comparison.

The graph depicts that the first class does not affect the travel time, as a result that the driver tends to eliminate all the negative facts by respecting all recommendations predefined. It's helped to maintain a smooth traffic, avoiding all undesirable and unplanned events.

Similarly the same thing, when we describe, respectively the Fig. 10,11, 12 mentioning the metrics of traffics mobility, particularly the total and the average waiting time, and the average of vehicular travel length, the results reveal that our system performed better than the classical one, which proves and demonstrates the effeteness of our solution.
The Fig. 13 shows the analysis of the observed vehicles' consumption results depending on density, the graph confirms that a fuel saving consumption is directly proportional and has an affinity with congestion road. Nevertheless, related to our approach, the fuel consumption product by V2IoT is much lower when compared to the normal communications. It's exactly the same thing happened to $\mathrm{CO} 2$ emissions, these emissions created by burning fuel are directly related to fuel consumption rate. That said, when the percentage of fuel increased, the emissions of $\mathrm{CO} 2$ realize a dramatically expanded and vice versa.

Furthermore, in congestion situation the normal vehicular communications produce negative effects into the traffic's performance of all metrics. In figures when we defined the rest of pollutant emissions, $P M, N O_{x}, \mathrm{CO}, \mathrm{CH}$, and the noise level emissions.

These traditional vehicular communications anchor the highest rate, the emissions are influenced by the augmentations of vehicles. On the other hand the V2IoT takes the lead with a mixture of the rise and stability.

In summary, the validation experiments expose that our approach offer the best performances between the mobility and emission metrics in the scope of scenario conditions analyzed. In the light of the experimental results we can conclude that in terms of the performance of V2IoT used in this work, the technique proposed, take the lead compared with the standard communications. We can clearly observe that the proposed dynamic traffic lights improve the intelligent transportation system, offering the opportunity to exploit the terms of smart mobility with a contribution for more efficient traffic and cleaner environment.

\section{CONCLUSIONS}

This paper depicts a part of the approach entitled V2IoT, which aims to use the techniques of Internet of Things to improve the famous concept of Intelligent Transportation System based on VANET communications. The ultimate goal is to offer a dynamic traffic lights algorithm, which controls the traffic lights by using the concept of calculating the vehicular density inspired by the Kerner three phase traffic theory. A straight path, to contribute for more efficient traffic and greener environment, among the highlights factors influencing of the concept of Smart Cities. That being said, a fundamental construct of smartness. In this sense, a quick glance in the experiments depicted, show that the results of V2IoT outperform with better mobility and emission metrics than the standard communications. Furthermore, the suggested model marks acceptable benefits in terms of service quality and optimization provisioning in vehicular networks. As future work, we plan to design or optimize a routing protocol inspired by the approach, while ensuring a good performance of network metrics in addition to metrics of mobility and emissions. Likewise, we are presently extending our experiments with a larger urban zone VANET instances.

\section{REFERENCES}

[1] T. Nam and T. A. Pardo, "Conceptualizing smart city with dimensions of technology, people, and institutions," in Proceedings of the 12th Annual International Digital Government Research Conference: Digital Government Innovation in Challenging Times, 2011, pp. 282-291.

[2] J. Gubbi, R. Buyya, S. Marusic, and M. Palaniswami, "Internet of Things (IoT): A vision, architectural elements, and future directions," Future Gener. Comput. Syst., vol. 29, no. 7, pp. 1645-1660, 2013. 
[3] L. Atzori, A. Iera, and G. Morabito, "The internet of things: A survey," Comput. Netw., vol. 54, no. 15, pp. 2787-2805, 2010.

[4] A. Rghioui, M. Bouhorma, and A. Benslimane, "Analytical study of security aspects in 6LoWPAN networks," in 2013 5th International Conference on Information and Communication Technology for the Muslim World (ICT4M), 2013, pp. 1-5.

[5] "Internet of Things (IoT) - Cisco IoT Product Portfolio Cisco." [Online]. Available: http://www.cisco.com/c/en/us/solutions/internet-ofthings/iot-products.html. [Accessed: 29-Apr-2016].

[6] T. Vaa, M. Penttinen, and I. Spyropoulou, "Intelligent transport systems and effects on road traffic accidents: state of the art," IET Intell. Transp. Syst., vol. 1, no. 2, pp. 81-88, Jun. 2007.

[7] H. Hartenstein and K. Laberteaux, VANET: vehicular applications and inter-networking technologies, vol. 1 . Wiley Online Library, 2010.

[8] S. Yousefi, M. S. Mousavi, and M. Fathy, "Vehicular ad hoc networks (VANETs): challenges and perspectives," in ITS Telecommunications Proceedings, 2006 6th International Conference on, 2006, pp. 761-766.

[9] Y. Hernafi, M. Bouhorma, M. B. Ahmed, A. Boudhir, and Y. A. Alluhaidan, "Clustering Architecture for Smart Mobility Control: Application to Pilgrims in the Great Mosque in El Hajj," J. Theor. Appl. Inf. Technol., vol. 78 , no. 1, p. 84, 2015.
[10] J. Santa, A. F. Gómez-Skarmeta, and M. SánchezArtigas, "Architecture and evaluation of a unified V2V and V2I communication system based on cellular networks," Comput. Commun., vol. 31, no. 12, pp. 2850-2861, 2008.

[11] "50\% Rise in Gridlock Costs by 2030 | Centre for Economics and Business Research." [Online]. Available: http://www.cebr.com/reports/the-future-economic-andenvironmental-costs-of-gridlock/. [Accessed: 29-Apr2016].

[12] "Touring Corporate - Presse - Enquête Touring: 3 fois plus de $\mathrm{CO} 2$ à cause de feux mal synchronisés." [Online]. Available: https://www.touring.be/fr/corporatesite/qui-sommesnous/presse/communiques/public-policy/plus-co2-parfeux-mal-synchronises/index.asp. [Accessed: 29-Apr2016].

[13] "Criticism of three-phase traffic theory." [Online]. Available: http://www.sciencedirect.com/science/article/pii/S01912 61509000228. [Accessed: 29-Apr-2016].

[14] B. S. Kerner, "Three-phase traffic theory and highway capacity," Phys. Stat. Mech. Its Appl., vol. 333, pp. 379440, 2004. 